Trend of Mean HIV Positives diagnosed during active index testing vs passive index testing periods

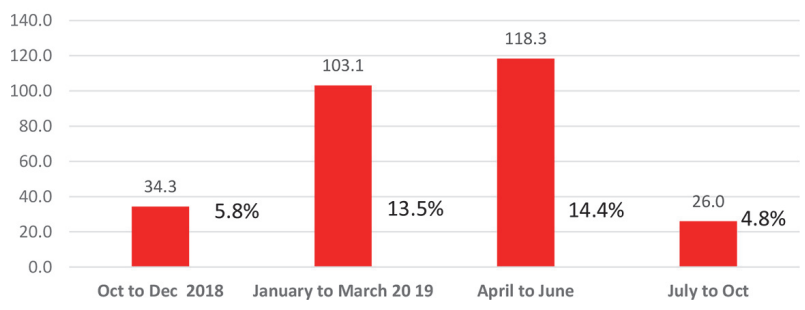

Abstract 12 Figure 2 Trend of Mean HIV Positives diagnosed during active index testing vs passive index

suboptimal with significant performance gaps in the Malawi Defence Force health facilities. We adopted active index testing in 7 facilities aiming at addressing the performance gap.

Objectives We sought to assess impact of index testing on overall HIV yield and explore factors associated with progress of active index testing.

Methods We conducted bottleneck analysis using problem tree analysis to identify root cause for low HIV yield performance. Using Plan Do Study Act (PDSA) approach, index testing was implemented using HIV diagnostic assistants (HDAs) for six months. Index testing program data was collected by testing modality weekly and run charts were plotted to study performance of HIV yield pre, during and post active index testing periods. In-depth interviews were also conducted with 10 HDAs from 7 facilities involved in the project to understand factors associated with index testing performance.

Results The mean HIV yield during passive index testing was 34.4 (6\%yield) and 26 (5\% yield) for the October-December 2018 and July-September 2019 quarters respectively. Active index testing mean yields were $103.1(13 \%$ yield) and 118 (14\% yield) for January-March and April-June 2019 quarters respectively. There was a significant rise in mean yield from $34.4(6 \%)$ to $103.1(13 \%$ yield) when index testing was implemented. There was a significant drop in mean yield from $118(14 \%$ yield $)$ to 26 (5\% yield) when active index testing was stopped. Table 1 provision of operational support for index testing implementation and dedicated monitoring of index testing activities (figures 1 and 2) through weekly data reviews were cited as the contributors to the index testing performances.

Conclusions Active index testing is a plausible approach towards accelerated HIV case finding and linkage into care.

\section{TRIALISTS VIEWS ON SUSTAINING, SPREADING AND SCALING-UP QUALITY IMPROVEMENT INTERVENTIONS}

${ }^{1}$ Celia Laur, ${ }^{2}$ Ann Marie Corrado, Jeremy Grimshaw, ${ }^{4}$ Noah Ivers. ${ }^{3}$ Women's College Hospital Institute for Health System Solutions and Virtual Care, and Women's College Research Institute, Women's College Hospital, Toronto, Ontario; ${ }^{2}$ The Peter Gilgan Centre for Women's Cancers, Women's College Hospital, Toronto, ON, Canada; ${ }^{3}$ Clinical Epidemiology Program, Ottawa Hospital Research Institute, Ottawa; and the Department of Medicine, University of Ottawa, Ottawa, ON, Canada; ${ }^{4}$ Women's College Hospital Institute for Health System Solutions and Virtual Care, and Women's College Research Institute, Women's College Hospital, Toronto, Ontario; and Department of Family and Community Medicine and Institute of Health Policy, Management and Evaluation, University of Toronto, Toronto, ON, Canada

10.1136/bmjoq-2020-IHI.13
Background Quality Improvement (QI) evaluations rarely consider how a successful intervention can be sustained, nor how to spread or scale to other locations. A survey of authors of randomized trials of diabetes QI interventions included in an ongoing systematic review found that $78 \%$ of trials reported improved quality of care, but $40 \%$ of these trials were not sustained.

Objectives To explore why and how the effective interventions were sustained, spread or scaled.

Methods A qualitative approach was used, focusing on case examples. Diabetes QI program trial authors were purposefully sampled and recruited for interviews. Authors were eligible if they had completed the survey, agreed to follow-up, and had a completed a diabetes QI trial they deemed 'effective'. Snowball sampling was used if the participant indicated someone could provide a different perspective on the same trial. Interviews were transcribed verbatim. Inductive thematic analysis was conducted to identify barriers and facilitators to sustainability, spread, and/or scale of the QI program, using case examples to show trajectories across projects and people.

Results Eleven of 44 eligible trialist participated. Four reported that the intervention was 'sustained' and nine were 'spread,' however interviews highlighted that these terms were interpreted differently over time. Participant stories highlighted the trajectories of how projects evolved and how research careers adapted to increase impact. Three interacting themes were identified: i) understanding the concepts of implementation, sustainability, spread and scale; ii) having the appropriate competencies; and iii) the need for individual, organisational and system capacity.

Conclusions Trialists need to think beyond local effectiveness to achieve population-level impact. Early consideration of whether an intervention is feasible and sustainable once research funding ends is necessary to plan for sustainability, spread and/or scale of effective QI programs. The competencies required for these goals are distinct from those required to implement or evaluate QI programs.

\section{THE COMMUNITY TRANSFORMATION MAP: TRACKING AND PLANNING COALITION-LEAD COMMUNITY CHANGE}

${ }^{1}$ Ariel Domlyn, ${ }^{2}$ Rohit Ramaswamy, ${ }^{3}$ Niñon Lewis, ${ }^{3}$ Marianne E McPherson. ${ }^{1}$ University of South Carolina, United Sates; ${ }^{2}$ University of North Carolina Chapel Hill, USA; ${ }^{3}$ Institute for Healthcare Improvement, USA

\subsection{6/bmjoq-2020-|HI.14}

Background Coalitions are promising structures for tackling health inequities. Supporting community health calls for selfadministered tools where coalitions can measure their capabilities, determine priorities, set goals, and assess progress. Maturity models map detailed, sequential stages of idealized progress. The Community Transformation Map (CTM) is maturity model where users self-assess and chart a trajectory for improvement. We describe the development, application, and evaluation of the CTM, a collaborative planning tool developed within the 100 Million Healthier Lives community transformation initiative.

Objectives We will (1) describe the theoretical and methodological basis for the CTM, (2) present CTM content, 
administration, and scoring process, (3) highlight lessons learned and strategies for practical application.

Methods Iterative, participatory action methods guided CTM development and use. The 40-item CTM captures three components of community transformation: Improvement, relationships, and equity. Eighteen coalitions used the CTM at four time-points, selecting areas for improvement deemed salient to their context. Each created action plans to address these areas. Ten semi-structured interviews assessed CTM use and contextual validity.

Results Coalition's CTM scores were averaged across three community transformation dimensions. This revealed wide variation in scores with context-specific strengths and weaknesses; with the exception of one outlier, no coalitions displayed consistent strengths across dimensions. Interviews revealed the CTM used as intended: collaboratively, inclusively, and for strategizing improvements. Users perceived the CTM's greatest value as a needs assessment that operationalized systemic change concepts into descriptive indicators.

Conclusions The CTM is an actionable collaboration tool for coalitions that has recently been adapted into a validated selfassessment for communities to understand improvements in health and equity. As the first translation of maturity models for coalition use, the CTM represents a promising structure for user-led, community-level planning.

\section{OVERCOMING STRUCTURAL INEQUITY IN HEALTH CARE CONTEXTS FOR PEOPLE LIVING WITH MENTAL ILLNESS AND SUBSTANCE USE ISSUES}

Thomas Ungar. Mental Health Commission of Canada, Canada

\subsection{6/bmjoq-2020-IHI.15}

Background Structural stigma arbitrarily limits opportunities and/or constrains the rights of people living with substance use issues and mental illnesses. It is difficult to see -because structural stigma, inequity, and systemic bias 'hides in plain sight,' and because we are just starting to develop measures and undertake research needed to bring structural stigma properly out into the open.

Objectives This presentation will describe the multiple layers of stigma as an inequity impacting quality care for people living with mental illness and substance use issues. It will also identify opportunities for interventions to improve and dismantle structural inequities within health-care organizations summarized in our framework to action.

Methods To better understand its effects and impacts, the Mental Health Commission of Canada Research Team conducted a comprehensive literature review, qualitative research among people with lived experience, and a promising partners report to identify key priorities and areas of focus for structural change in the health-care system.

Results Early findings demonstrate that addressing structural stigma in health-care contexts requires a multipronged approach, working towards cultural change by improving attitudes and practices of health-care practitioners, strengthening integration and coordination of care, using a stigma-informed lens to evaluate and revise policies and practices, prioritizing the meaningful inclusion of people with lived and living experience at all levels, especially within research, policy and

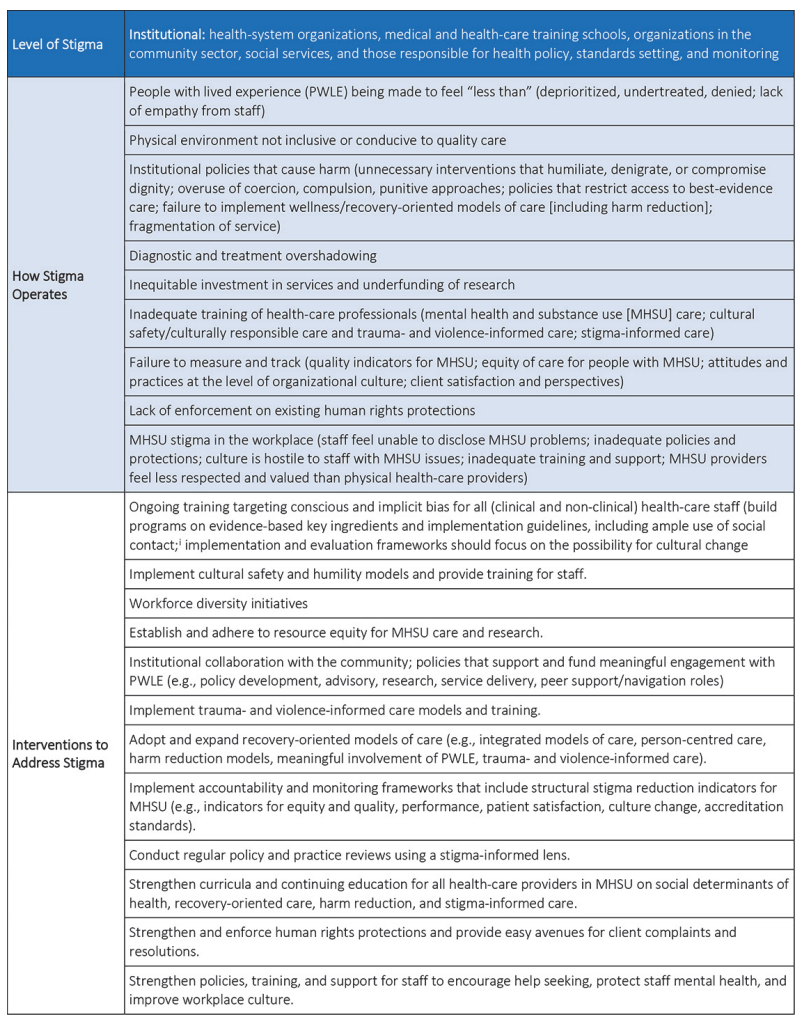

Abstract 15 Figure 1 Combating mental illness- and substance userelated structural stigma in healthcare: A framework for action

\begin{tabular}{|c|c|}
\hline Level of Stigma & $\begin{array}{l}\text { Institutional: health-yystem organizations, medical and health-care training schools, organizations in the } \\
\text { community sector, social services, and those responsible for health policy, standards setting, and monitoring }\end{array}$ \\
\hline \multirow{12}{*}{$\begin{array}{l}\text { Potential } \\
\text { Outcomes }\end{array}$} & An institutional environment that is inclusive, welcoming, diverse, and safe \\
\hline & Organizations that can meet the needs of all populations, including PWLE \\
\hline & A reduction in stigmatizing beliefs and attitudes among staff and across the organization \\
\hline & Improved patient/client ratings of care, satisfaction, and trust \\
\hline & Improved patient/client outcomes (physical and mental health for PWLE; quality of life for PWLE) \\
\hline & Earlier engagement in care for PWLE due to earlier help seeking \\
\hline & Better retention in care and treatment for PWLE \\
\hline & More appropriate and best-evidence care provided to PWLE \\
\hline & Greater compassion satisfaction among staff \\
\hline & Improved mental health of health-care staff \\
\hline & Less time off work; improved worker retention \\
\hline & MHSU providers that feel valued and equitably compensated within the health-care system \\
\hline
\end{tabular}

Abstract 15 Figure 2 Adapted from "Table 2 - Action Framework for Building an Inclusive Health System," by the Public Health Agency of Canada, Addressing stigma: Towards a more inclusive health system, The chief public health officer's report on the state of public health in Canada (p. 41), 2019, Ottawa, Canada: Copyright 2019 by Her Majesty the Queen in Right of Canada, as represented by the Minister of Health

service delivery, and establishing mechanisms to monitor structural stigma (figures 1 and 2).

Conclusions This research brought into focus a comprehensive picture of the problem of structural stigma inequity; how it is experienced, how it impacts on health and quality of life outcomes, and important strategies and approaches for reshaping the way health service delivery and care is provided for people with substance use and mental health problems. These include sharing innovative models of care, developing training modules, and developing measures to identify structural stigma. 